\title{
Synthesis and characterization of tert-butylcyclopentadienyl derivatives of hafnium
}

\author{
Tomás Cuenca, Cristina Montejano, Pascual Royo * \\ Departamento de Química Inorgánica. Universidad de Alcalá de Henares, Campus Universitario, 28871-Alcalá de Henares, Spain
}

Received 3 August 1995

\begin{abstract}
Reaction of $\mathrm{Si}\left(\mathrm{C}_{5} \mathrm{H}_{3}-1,3-{ }^{\prime} \mathrm{Bu}_{2}\right) \mathrm{Me}_{3}$ with $\mathrm{HfCl}_{4}$ in toluene or hexane at room temperature afforded the new trichloro monocyclopentadienyl derivatives $\left[\mathrm{Hf}\left(\eta^{5}-\mathrm{C}_{5} \mathrm{H}_{3}-1,3-\mathrm{Bu}_{2}\right) \mathrm{Cl}_{3}\right]$ (1). Treatment of $\left[\mathrm{Hf}\left(\eta^{5}-\mathrm{C}_{5} \mathrm{H}_{3}-1,3-{ }^{\prime} \mathrm{Bu}_{2}\right) \mathrm{Cl}_{3}\right]$ with the appropriate alkylating reagent in hexane gave the mono[1,3-di(tert-butyl)cyclopentadienyl] trialkyl hafnium complexes [Hf $\left.\left(\eta^{5}-\mathrm{C}_{5} \mathrm{H}_{3}-1,3-\mathrm{Bu}_{2}\right) \mathrm{R}_{3}\right]\left[\mathbf{R}=\mathrm{CH}_{3}(2)\right.$,

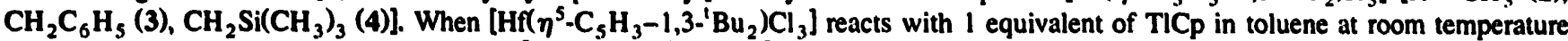
the mixed dicyclopentadienyl complex $\left[\mathrm{HIf}\left(\eta^{5} \cdot \mathrm{C}_{5} \mathrm{H}_{3}-1,3-{ }^{\prime} \mathrm{Bu}_{2}\right)\left(\eta^{5} \cdot \mathrm{C}_{5} \mathrm{H}_{5}\right) \mathrm{Cl}_{2}\right](5)$ was isolated in almost quantitative yield. Reaction of 5 with LiMe led to the dimethyl complex $\left[\mathrm{Hf}\left(\eta^{5}-\mathrm{C}_{5} \mathrm{H}_{3}-1,3-\mathrm{Bu}_{2}\right)\left(\eta^{5}-\mathrm{C}_{5} \mathrm{H}_{5}\right)\left(\mathrm{CH}_{3}\right)_{2}\right](6)$. However, the analogous reaction of a hexane solution of 5 with $\mathrm{MgBz}_{2} \cdot 2 \mathrm{THF}$ in the presence of traces of water afforded the $\mu$ oxo derivative $\left[\left(\mathrm{Hf}\left(\eta^{5} \cdot \mathrm{C}_{5} \mathrm{H}_{3}-1,3-^{\prime} \mathrm{Bu}_{2}\right)\left(\eta^{5}\right.\right.\right.$. $\left.\left.\left.\mathrm{C}_{5} \mathrm{H}_{5}\right)\left(\mathrm{CH}_{2} \mathrm{C}_{6} \mathrm{H}_{5}\right)\right)_{2}(\mu-\mathrm{O})\right](7)$.
\end{abstract}

Keywords: Hafnium; Cyclopentadienyls; Preparation

\section{Introduction}

$\eta^{5}$-Cyclopentadienyl derivatives of transition metals have played an important role in structural, synthetic and catalytic organometallic chemistry. Replacement of one or more of the cyclopentadienyl ring hydrogens by alkyl groups led to a large variety of substituted cyclopentadienyl ligands [1]. This effect modifies the steric and electronic properties of the metal centre and implies important changes in the structural and chemical behaviour of this type of compound. Several titanium, zirconium and hafnium complexes containing functionalized cyclopentadienyl ligands have been described $[1,2]$, but only a few examples of tert-butylcyclopentadienyl derivatives of these elements are known [3].

The presence of the tert-butyl substituents in the cyclopentadienyl ring introduces significant changes, increasing the solubility of the complexes and providing easily recognizable ${ }^{1} \mathrm{H}$ and ${ }^{13} \mathrm{C}$ NMR resonances. These properties make the 1,3-di(tert-butyl)cyclopentadienyl ring a good system to synthesize soluble monocyclopentadienyl titanium, zirconium and hafnium complexes and to study their structural and chemical behaviour.

\footnotetext{
- Corresponding author.
}

In this paper we report the synthesis and characterization of new 1,3-di(tert-butyl)monocyclopentadienyl hafnium complexes [ $\left.\mathrm{Hf}\left(\eta^{5}-\mathrm{C}_{5} \mathrm{H}_{3}-1,3{ }^{\prime} \mathrm{Bu}_{2}\right) \mathrm{Cl}_{3}\right](1)$ and $\left[\mathrm{Hf}\left(\eta^{5}-\mathrm{C}_{5} \mathrm{H}_{3}-1,3-\mathrm{Bu}_{2}\right) \mathrm{R}_{3}\right]\left[\mathrm{R}=\mathrm{CH}_{3}(2), \mathrm{CH}_{2} \mathrm{C}_{6} \mathrm{H}_{5}\right.$ (3), $\left.\mathrm{CH}_{2} \mathrm{Si}\left(\mathrm{CH}_{3}\right)_{2}(4)\right]$ and the mixed cyclopentadienyl derivatives [ $\left.\mathrm{Hf}\left(\eta^{5}-\mathrm{C}_{5} \mathrm{H}_{3}-1,3 \cdot \cdot^{\prime} \mathrm{Bu} \mathrm{u}_{2}\right)\left(\eta^{5}-\mathrm{C}_{5} \mathrm{H}_{5}\right) \mathrm{Cl}_{2}\right]$ (5). [Hf $\left(\eta^{5}-\mathrm{C}_{5} \mathrm{H}_{3}-1,3-\mathrm{Bu}_{2}\right)\left(\eta^{5}-\mathrm{C}_{5} \mathrm{H}_{5}\right)\left(\mathrm{CH}_{3}\right)_{2}$ ] (6) and $\left[\left(\mathrm{Hf}^{2}\left(\eta^{5} \cdot \mathrm{C}_{5} \mathrm{H}_{3}-1,3-\mathrm{Bu}_{2}\right)\left(\eta^{5}-\mathrm{C}_{5} \mathrm{H}_{5}\right)\left(\mathrm{CH}_{2} \mathrm{C}_{6} \mathrm{H}_{5}\right)\right]_{2}(\mu-\mathrm{O})\right]$ (7). All these compounds were characterized by analytical and spectroscopic methods.

\section{Discussion of synthetic and spectroscopic results}

We have prepared compound $\left[\mathrm{Hf}\left(\eta^{5}-\mathrm{C}_{5} \mathrm{H}_{3}-1,3-\right.\right.$ $\left.\left.{ }^{\prime} \mathrm{Bu}_{2}\right) \mathrm{Cl}_{3}\right](1)$ by treatment of pure anhydrous $\mathrm{HfCl}_{4}$ with $\left.\mathrm{Si}\left(\eta^{5}-\mathrm{C}_{5} \mathrm{H}_{3}-1,3\right)^{\prime} \mathrm{Bu}_{2}\right) \mathrm{Me}_{3}$ in toluene or heptane at $80^{\circ} \mathrm{C}$, a well known and routine technique. The trimethylsilyl 1,3-di(tert-butyl)cyclopentadiene reagent was obtained by reaction of 1,3-di(tert-butyl)cyclopentadiene, synthesized as a mixture of different possible isomers, with Lin-Bu and chlorotrimethylsilane [4]. When a hexane solution of complex 1 was treated with a diethyl ether solution of $\mathrm{MgClMe}$, in a molar ratio $1: 3.1$, an almost quantitative yield of the trimethyl 
hafnium compound [Hf( $\left.\eta^{5}-\mathrm{C}_{5} \mathrm{H}_{3}-1,3-\mathrm{Bu}_{2}\right) \mathrm{Me}_{3}$ ] (2) was isolated after filtration and evaporation of the solvent. The same reaction of the trichloro hafnium complex 1 with $\mathrm{MgBz}_{2} \cdot 2 \mathrm{THF}$ afforded the tribenzyl complex $\left[\mathrm{Hf}\left(\eta^{5}-\mathrm{C}_{5} \mathrm{H}_{3}-1,33^{\prime} \mathrm{Bu}_{2}\right)\left(\mathrm{CH}_{2} \mathrm{C}_{6} \mathrm{H}_{5}\right)_{3}\right](3)$, and reaction of 1 with $\mathrm{LiCH}_{2} \mathrm{SiMe}_{3}$ led to the trialkyl derivative $\left[\mathrm{Hf}\left(\eta^{5}-\mathrm{C}_{5} \mathrm{H}_{3}-1,3-\mathrm{Bu}_{2}\right)\left(\mathrm{CH}_{2} \mathrm{SiMe}_{3}\right)_{3}\right](4)$ (Scheme 1).

The mixed dicyclopentadienyl compound $\left[\mathrm{Hf}\left(\eta^{5}\right.\right.$ $\left.\left.\mathrm{C}_{5} \mathrm{H}_{3}-1,3-\mathrm{Bu}_{2}\right)\left(\eta^{5}-\mathrm{C}_{5} \mathrm{H}_{5}\right) \mathrm{Cl}_{2}\right]$ (5) can be prepared, as a white solid in $95 \%$ yield, by treatment of 1 with TICp in an equimolar ratio in toluene under reflux. Reaction of a diethyl ether solution of 5 with LiMe (molar ratio 1:2.1) gave the dimethyl complex [ $\mathrm{Hf}\left(\eta^{5}-\mathrm{C}_{5} \mathrm{H}_{3}-1,3-\right.$ $\left.\left.{ }^{\prime} \mathrm{Bu}_{2}\right)\left(\mathrm{C}_{5} \mathrm{H}_{5}\right)\left(\mathrm{CH}_{3}\right)_{2}\right](6)$. Formation of the analogous dibenzyl complex was observed when the reaction of a hexane solution of 5 with $\mathbf{M g B z}_{2} \cdot 2$ THF was carried out under rigorously anhydrous conditions into a drybox. However, this compound was extremely moisture sensitive and was not isolated as a pure solid. The presence of traces of water in the reaction mixture led to its transformation into the $\mu$-oxo derivative [ $\left[\mathrm{Hf}\left(\eta^{5}\right.\right.$ $\left.\left.\left.\mathrm{C}_{5} \mathrm{H}_{3}-1,3-^{\prime} \mathrm{Bu}_{2}\right)\left(\eta^{5}-\mathrm{C}_{5} \mathrm{H}_{5}\right)\left(\mathrm{CH}_{2} \mathrm{C}_{6} \mathrm{H}_{5}\right)\right\}_{2}(\mu-\mathrm{O})\right]$ (7), which was quantitatively fonned as a yellow microcrystalline solid when wet hexane was used as solvent.

Complexes 1-6 were isolated as brown or yellow oils or microcrystalline solids, very soluble in all common, even aliphatic, solvents. They are air and moisture sensitive but may be stored unchanged for months under an inert atmosphere (dinitrogen or argon), 1 and 5 being the most stable.
${ }^{1} H$ NMR spectra of compounds 1-6 show the expected resonances for the alkyl groups bonded to the metal atom (see Experimental section). The tert-butyl protons appear as a singlet and the cyclopentadienyl protons of the $\mathrm{C}_{5} \mathrm{H}_{3}{ }^{\prime} \mathrm{Bu}_{2}$ ring as one pseudodoublet (2H) and one pseudotriplet (1H) corresponding to an AA'B spin system, whereas the $\mathrm{C}_{5} \mathrm{H}_{5}$ protons give one singlet. The ${ }^{1} \mathrm{H}$ NMR spectrum for compound 7 shows two singlets $(\delta 1.05, \delta 1.15)$ for the tert-butyl protons and two sets of doublets $(\delta 2.19,2.20)$ for the diastereotopic methylene protons of the benzyl group. The cyclopentadieny 1 protonis exinibit one singlet at $\delta 5.85$ for the $\mathrm{C}_{5} \mathrm{H}_{5}$ ring protons and three multiplets $(\delta 5.59$, $5.99,6.00$ ) for the protons of the $\mathrm{C}_{5} \mathrm{H}_{3}{ }^{\prime} \mathrm{Bu}_{2}$ ring corresponding to an $\mathrm{ABC}$ spin system.

\section{Experimental}

All manipulations were performed under an inert atmosphere (dinitrogen or argon) using Schlenk and high vacuum line techniques or a VAC glove-box Model HE 63P. Solvents were purified by distillation from an appropriate drying/deoxygenating agent (sodium for toluene, sodium/benzophenone for diethyl ether and sodium/potassium alloy for hexane). LiMe (1.6 M diethyl ether solution), $\mathrm{MgClMe}$ ( $3 \mathrm{M}$ diethyl ether solution) and $\mathrm{HfCl}_{4}$ (Aldrich) were purchased and used without further purification. $\mathrm{Si}\left(\mathrm{C}_{5} \mathrm{H}_{3}-1,3-\mathrm{Bu}_{2}\right) \mathrm{Me}_{3}$ [4], $\mathrm{MgBz}_{2} \cdot 2 \mathrm{THF}$ [5], $\mathrm{LiCH}_{2} \mathrm{SiMe}_{3}[6]$ and $\mathrm{TlCp}$ [7] were

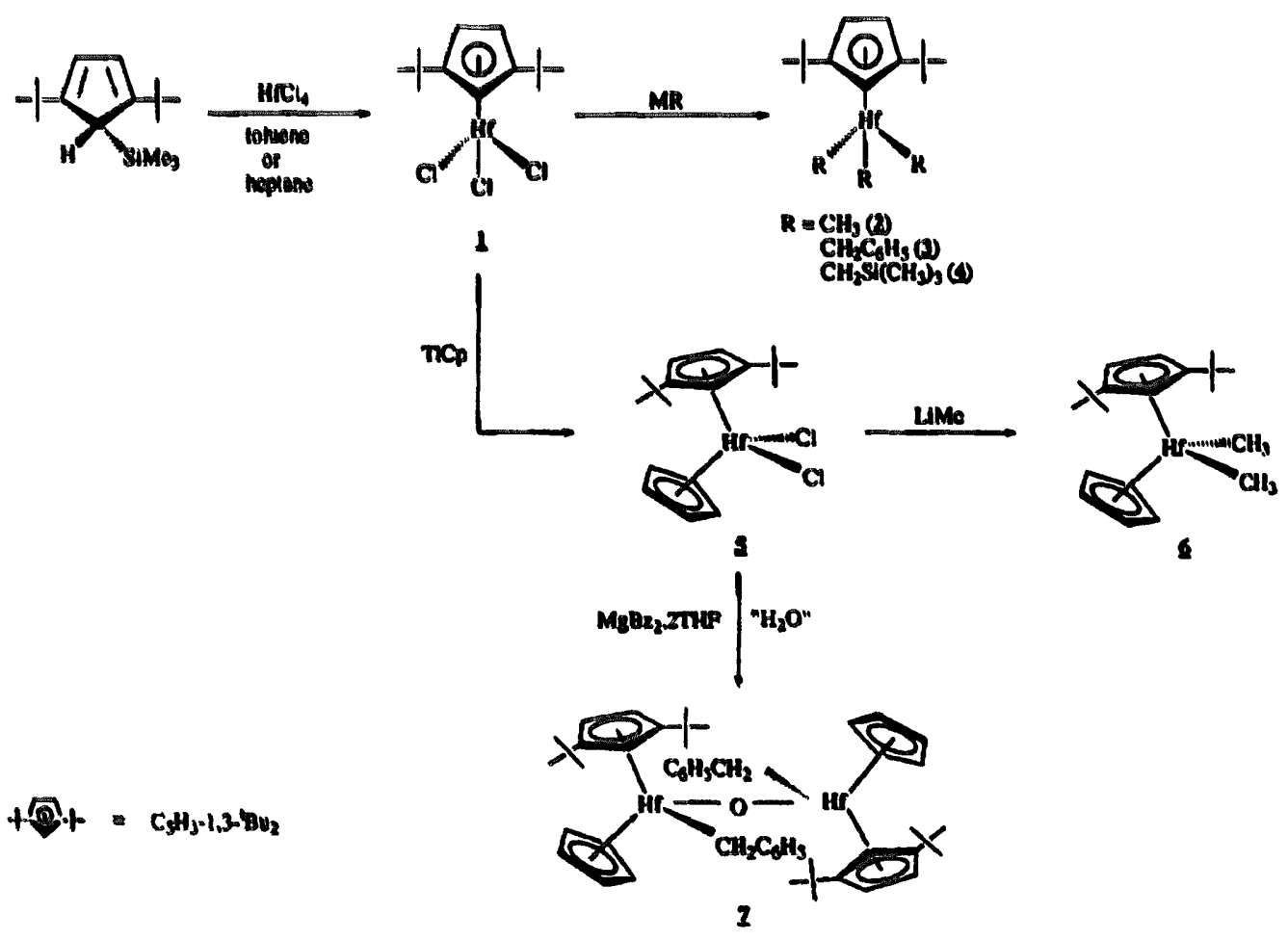

Scheme 1. 
prepared according to literature procedures. NMR spectra were recorded on Varian FT-80 and Varian Unity FT-300 instruments $\left({ }^{1} \mathrm{H}\right.$ and ${ }^{13} \mathrm{C}$ chemical shifts were referenced to $\left.\mathrm{SiMe}_{4}, \delta=0 \mathrm{ppm}\right)$. Mass spectra were recorded on a Hewlett Packard 5890 spectrometer. Elemental $\mathbf{C}$ and $\mathbf{H}$ analyses were carried out on a PerkinElmer 240B microanalyzer.

\subsection{Preparation of $\left[\mathrm{H} f\left(\mathrm{C}_{5} \mathrm{H}_{3}-1,3-\mathrm{Bu}_{2}\right) \mathrm{Cl}_{3}\right]$ (1)}

$\mathrm{Si}\left(\mathrm{C}_{5} \mathrm{H}_{3}-1,3-{ }^{-} \mathrm{Bu}_{2}\right) \mathrm{Me}_{3}(2.96 \mathrm{ml}, 2.34 \mathrm{~g}, 9.36 \mathrm{mmol})$ was added, at $0^{\circ} \mathrm{C}$ and under argon, to a solution of $\mathrm{HfCl}_{4}(3.0 \mathrm{~g}, 9.36 \mathrm{mmnl})$ in toluene $(100 \mathrm{ml})$. The reaction mixture was slowly warmed to room temperature and then under reflux for $24 \mathrm{~h}$ to give a red-brown solution. The resulting solution was filtered and the solvent was evaporated under vacuum to obtain a brown solid which was recrystallized from toluene/hexane and characterized as 1 ( $3.15 \mathrm{~g}, 74 \%$ yield).

Anal. Found: $\mathrm{C}, 33.91 ; \mathrm{H}, 4.62 . \mathrm{C}_{13} \mathrm{H}_{21} \mathrm{HfCl}_{3}$. Calc.: C, 33.76; H, 4.54\%. ${ }^{1} \mathrm{H}$ NMR ( $\left.300 \mathrm{MHz}, \mathrm{C}_{6} \mathrm{D}_{6}, 25^{\circ} \mathrm{C}\right)$ : $\delta 1.12\left(\mathrm{~s}, 18 \mathrm{H},{ }^{\prime} \mathrm{Bu}\right), 5.87\left(\mathrm{~d}, 2 \mathrm{H}, \mathrm{C}_{5} \mathrm{H}_{3}\right), 6.32(\mathrm{t}, 1 \mathrm{H}$, $\left.\mathrm{C}_{5} \mathrm{H}_{3}\right) .{ }^{13} \mathrm{C}$ NMR (75 MHz, $\left.\mathrm{C}_{6} \mathrm{D}_{6}, 25^{\circ} \mathrm{C}\right): \delta 31.8$ ('Bu), $32.1\left(\mathrm{C}_{\text {ipso }}\right.$ 'Bu), 103.1, 104.1 $\left(\mathrm{C}_{5} \mathrm{H}_{3}\right), 143.3$ $\left(\mathrm{C}_{\text {ipso }} \mathrm{C}_{5} \mathrm{H}_{3}\right)$. Electron impact mass spectrum $(70 \mathrm{eV})$ : $m / z=462(14 \%)[\mathrm{M}]^{+} ; \quad m / z=447$ (100\%) [M$\left.\mathrm{CH}_{3}\right]^{+}$.

\subsection{Preparation of $\left[\mathrm{Hf}\left(\mathrm{C}_{5} \mathrm{H}_{3}-1,3-^{\prime} \mathrm{Bu} \mathrm{u}_{2}\right)\left(\mathrm{CH}_{3}\right)_{3}\right]$}

A $3 \mathrm{M}$ solution of $\mathrm{MgClMe}$ in tetrahydrofuran (1.12 $\mathrm{ml}, 336 \mathrm{mmol}$ ) was added to a hexane solution $(50 \mathrm{ml})$ containing $\mathrm{Hf}\left(\mathrm{C}_{5} \mathrm{H}_{3}-1,3-\mathrm{Bu}_{2}\right) \mathrm{Cl}_{3}(0.5 \mathrm{~g}, 1.08 \mathrm{mmol})$ at $-78^{\circ} \mathrm{C}$. The reaction mixture was stirred for $3 \mathrm{~min}$ and then slowly warmed to room temperature and stirred for $48 \mathrm{~h}$. After filtration, the solvent was removed in vacuo to give a yellow oil which was recrystallized from toluene/hexane and characterized as $2(0.41 \mathrm{~g}$, $96 \%$ yield).

Anal. Found: $\mathrm{C}_{4}$ 48.41; $\mathrm{H}, 7.63 . \mathrm{C}_{16} \mathrm{H}_{30} \mathrm{Hf}$. Calc.: $\mathrm{C}$, 47.91; $\mathrm{H}, 7.48 \%$. $\mathrm{H}$ NMR (300 $\left.\mathrm{MHz}, \mathrm{C}_{6} \mathrm{D}_{6}, 25^{\circ} \mathrm{C}\right): \delta$ $1.11\left(\mathrm{~s}, 18 \mathrm{H},{ }^{\prime} \mathrm{Bu}\right), 0.33(\mathrm{~s}, 9 \mathrm{H}, \mathrm{Hf}-\mathrm{Me}), 5.68(\mathrm{~d}, 2 \mathrm{H}$, $\left.\mathrm{C}_{5} \mathrm{H}_{3}\right), 5.99\left(\mathrm{t}, 1 \mathrm{H}, \mathrm{C}_{5} \mathrm{H}_{3}\right) .{ }^{13} \mathrm{C}$ NMR (75 MHz, $\mathrm{C}_{6} \mathrm{D}_{6}$, $25^{\circ} \mathrm{C}$ ): $\delta 31.4$ ('Bu), $32.3\left(\mathrm{C}_{i p s o}{ }^{\mathrm{B}} \mathrm{Bu}\right), 57.3,\left(\mathrm{CH}_{3}\right)$, 103.0, 104.4 $\left(\mathrm{C}_{5} \mathrm{H}_{3}\right), 143.5\left(\mathrm{C}_{\text {ipso }} \mathrm{C}_{5} \mathrm{H}_{3}\right)$. Electron impact mass spectrum $(70 \mathrm{eV}): \mathrm{m} / z=397(70 \%)[\mathrm{M}-$ $\left.\mathrm{CH}_{3}\right]^{+}$.

\subsection{Preparation of $\left[\mathrm{Hf}\left(\mathrm{C}_{5} \mathrm{H}_{3}-1,3-\mathrm{Bu}_{2}\right)\left(\mathrm{CH}_{2} \mathrm{C}_{6} \mathrm{H}_{5}\right)_{3}\right]$} (3)

$1.1 \mathrm{~g}(3.11 \mathrm{mmol})$ of $\mathrm{MgBz}_{2} \cdot 2 \mathrm{THF}$ was added to a solution in hexane $(50 \mathrm{ml})$ containing $0.9 \mathrm{~g}(1.94 \mathrm{mmol})$ of $\left[\mathrm{Hf}\left(\mathrm{C}_{5} \mathrm{H}_{3}-1,3-\mathrm{Bu}_{2}\right) \mathrm{Cl}_{3}\right]$ and cooled to $-78^{\circ} \mathrm{C}$. The reaction mixture was slowly warmed to room temperature and stirred for $24 \mathrm{~h}$. After filtration, the resulting yellow solution was evaporated to dryness. Recrystal- lization from hexane at $-30^{\circ} \mathrm{C}$ gave a yellow microcrystalline solid which was characterized as $3(1.05 \mathrm{~g}$, $60 \%$ yield).

Anal. Found: C, 64.24; $\mathrm{H}, 7.1 . \mathrm{C}_{34} \mathrm{H}_{42} \mathrm{Hf}$. Calc.: $\mathrm{C}$, $64.1 ; \mathrm{H}, 7.2 \%$. ' $\mathrm{H}$ NMR (300 MHz, $\left.\mathrm{C}_{6} \mathrm{D}_{6}, 25^{\circ} \mathrm{C}\right): \delta$ $1.05\left(\mathrm{~s}, 18 \mathrm{H},{ }^{\prime} \mathrm{Bu}\right), 2.06\left(\mathrm{~s}, 6 \mathrm{H}, \mathrm{CH}_{2}\right), 5.54(\mathrm{~d}, 2 \mathrm{H}$, $\left.\mathrm{C}_{5} \mathrm{H}_{3}\right), 5.85\left(\mathrm{t}, 1 \mathrm{H}, \mathrm{C}_{5} \mathrm{H}_{3}\right), 6.81(\mathrm{~m}, \mathrm{o}-, \mathrm{p}-, \mathrm{m}-\mathrm{Ph}){ }^{1} \mathrm{H}$ NMR (300 $\left.\mathrm{MHz}, \mathrm{CDCl}_{3}, 2^{\circ} \mathrm{C}\right): \delta 1.17\left(\mathrm{~s}, 18 \mathrm{H},{ }^{\mathrm{t}} \mathrm{Bu}\right)$, $1.93\left(\mathrm{~s}, 6 \mathrm{H}, \mathrm{CH}_{2}\right), 5.70\left(\mathrm{~d}, 2 \mathrm{H}, \mathrm{C}_{5} \mathrm{H}_{3}\right), 6.06(\mathrm{t}, 1 \mathrm{H}$, $\left.\mathrm{C}_{5} \mathrm{H}_{3}\right), 6.65,7.12(\mathrm{~m}, \mathrm{Ph}) .{ }^{13} \mathrm{C} \mathrm{NMR}\left(75 \mathrm{MHz}, \mathrm{CDCl}_{3}\right.$, $\left.25^{\circ} \mathrm{C}\right)$ : $\delta 31.6\left({ }^{\mathrm{B} u}\right), 33.0\left(\mathrm{C}_{\text {ipso }}{ }^{\mathrm{B}} \mathrm{Bu}\right), 84.2\left(\mathrm{CH}_{2}\right)$, $105.4,106.8\left(\mathrm{C}_{5} \mathrm{H}_{3}\right), 122.4,127.3,128.4(\mathrm{Ph}), 146.2$ $\left(\mathrm{C}_{\text {ipso }} \mathrm{C}_{5} \mathrm{H}_{3}\right.$ ).

\subsection{Preparation of $\left[\mathrm{Hf}\left(\mathrm{C}_{5} \mathrm{H}_{3}-1,3-^{\prime} \mathrm{Bu}_{2}\right)\left(\mathrm{CH}_{2} \mathrm{SiMe}_{3}\right)_{3}\right]$} (4)

$0.34 \mathrm{~g}$ (3.3 mmol) of $\mathrm{LiCH}_{2} \mathrm{SiMe}_{3}$ was added to a solution in hexane $(50 \mathrm{ml})$ containing $0.5 \mathrm{~g}(1.08 \mathrm{mmol})$ of $\left[\mathrm{Hf}\left(\mathrm{C}_{5} \mathrm{H}_{3}-1,3-{ }^{\prime} \mathrm{Bu}_{2}\right) \mathrm{Cl}_{3}\right]$ and cooled to $-78^{\circ} \mathrm{C}$. The reaction mixture was slowly warmed to room temperature and stirred for $24 \mathrm{~h}$. After filtration, the resulting yellow solution was evaporated to dryness. Recrystallization from hexane at $-30^{\circ} \mathrm{C}$ gave a yellow microcrystalline solid which was characterized as $4(0.64 \mathrm{~g}$, 98\% yield).

Anal. Found: $\mathrm{C}, 48.21$; $\mathrm{H}, 9.0 . \mathrm{C}_{19} \mathrm{H}_{36} \mathrm{Si}_{3} \mathrm{Hf}$. Calc.: C, 48.62; H, 8.75\%. ' $\mathrm{H}$ NMR $\left(300 \mathrm{MHz}, \mathrm{C}_{6} \mathrm{D}_{6}, 25^{\circ} \mathrm{C}\right)$ : $\delta 0.13\left(\mathrm{~s}, 6 \mathrm{H}, \mathrm{CH}_{2}\right), 0.24\left(\mathrm{~s}, 27 \mathrm{H}, \mathrm{CH}_{3}-\mathrm{Si}\right), 1.18$ (s, $\left.18 \mathrm{H},{ }^{\prime} \mathrm{Bu}\right), 5.91\left(\mathrm{~d}, 2 \mathrm{H}, \mathrm{C}_{5} \mathrm{H}_{3}\right), 6.08\left(\mathrm{t}, 1 \mathrm{H}, \mathrm{C}_{5} \mathrm{H}_{3}\right){ }^{13} \mathrm{C}$ NMR (75 MHz, $\left.\mathrm{C}_{6} \mathrm{D}_{6}, 25^{\circ} \mathrm{C}\right): \delta 3.3\left(\mathrm{CH}_{3}-\mathrm{Si}\right), 32.0$ ('Bu), 32.7 ( $\mathrm{C}_{\text {ipso }}$ ' $\left.\mathrm{Bu}\right), 69.6\left(\mathrm{CH}_{2}\right), 104.4,104.5$ $\left(\mathrm{C}_{5} \mathrm{H}_{3}\right), 143.0\left(\mathrm{C}_{i p s o} \mathrm{C}_{5} \mathrm{H}_{3}\right)$.

3.5. Preparation of [ $\left.\left.\mathrm{Hf}^{\mathrm{C}} \mathrm{C}_{5} \mathrm{H}_{3}-1,3 \mathrm{-}^{\prime} \mathrm{Bu} \mathrm{u}_{2}\right)\left(\mathrm{C}_{5} \mathrm{H}_{5}\right) \mathrm{Cl}_{2}\right]$

$0.54 \mathrm{~g}(2.19 \mathrm{mmol})$ of freshly sublimed TICp were added at room temperature to a solution of $\left[\mathrm{Hf}\left(\mathrm{C}_{5} \mathrm{H}_{3}-\right.\right.$ $\left.\left.1,3 \mathrm{I}^{\prime} \mathrm{Bu}_{2}\right) \mathrm{Cl}_{3}\right](1.01 \mathrm{~g}, 2.19 \mathrm{mmol})$ in toluene $(100 \mathrm{ml})$. The reaction mixture was stirred under reflux for $24 \mathrm{~h}$ to give a yellow solution. After filtration, the solvent was evaporated under vacuum to afford a white solid which was recrystallized from toluene/hexane at $-30^{\circ} \mathrm{C}$ to give a microcrystalline compound characterized as 5 ( $1.06 \mathrm{~g}, 98 \%$ yield).

Anal. Found: $\mathrm{C}, 43.64 ; \mathrm{H}, 5.19 . \mathrm{C}_{18} \mathrm{H}_{26} \mathrm{HIfCl}_{2}$. Calc.: C, $43.97 ; \mathrm{H}, 5.28 \%$. ' $\mathrm{H}$ NMR $\left(300 \mathrm{MHz}, \mathrm{C}_{6} \mathrm{D}_{6}, 25^{\circ} \mathrm{C}\right)$ :

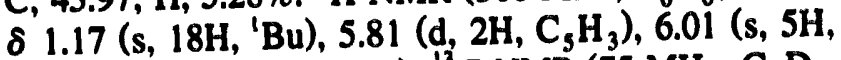
$\left.\mathrm{C}_{5} \mathrm{H}_{5}\right), 6.07\left(\mathrm{t}, 1 \mathrm{H}, \mathrm{C}_{5} \mathrm{H}_{3}\right) .{ }^{13} \mathrm{C}$ NMR $\left(75 \mathrm{MHz}, \mathrm{C}_{6} \mathrm{D}_{6}\right.$, $25^{\circ} \mathrm{C}$ ): $\delta 31.4$ ('Bu), 33.7 ( $\left.\mathrm{C}_{\text {ipso }}{ }^{\prime} \mathrm{Bu}\right), 110.9,111.1$ $\left(\mathrm{C}_{5} \mathrm{H}_{3}\right), 114.6\left(\mathrm{C}_{5} \mathrm{H}_{5}\right), 141.7\left(\mathrm{C}_{i p s o} \mathrm{C}_{5} \mathrm{H}_{3}\right)$.

3.6. Preparation of $\left[\mathrm{Hf}\left(\mathrm{C}_{5} \mathrm{H}_{3}-1,3-\mathrm{B}^{\prime} \mathrm{u}_{2}\right)\left(\mathrm{C}_{5} \mathrm{H}_{5}\right)\left(\mathrm{CH}_{3}\right)_{2}\right]$ (6)

A $1.6 \mathrm{M}$ solution of LiMe in diethyl ether $(0.80 \mathrm{ml}$, $1.28 \mathrm{mmol}$ ) was added to a diethyl ether solution (50 
m!) containing [ $\left.\mathrm{Hf}\left(\mathrm{C}_{5} \mathrm{H}_{3}-1,3-^{\prime} \mathrm{Bu}_{2}\right)\left(\mathrm{C}_{5} \mathrm{H}_{5}\right) \mathrm{Cl}_{2}\right](0.3 \mathrm{~g}$, $0.61 \mathrm{mmol})$ at $-78^{\circ} \mathrm{C}$. The reaction mixture was stirred for $30 \mathrm{~min}$ and then slowly warmed to room temperature and stirred for $\mathbf{3}$ h. After filtration, the solvent was removed in vacuo to give a yellow oil which was extracted into hexane. By cooling the resulting solution to $-30^{\circ} \mathrm{C}$ a microcrystalline yellow solid was obtained which was characterized as $6(0.23 \mathrm{~g}, 85 \%$ yield $)$.

Anal. Found: C, 52.93; $\mathrm{H}, 6.97$. $\mathrm{C}_{20} \mathrm{H}_{32} \mathrm{Hf}$. Calc.: $\mathrm{C}$, 53.25; H, 7.11\%. 'H NMR (300 MHz, $\left.\mathrm{C}_{6} \mathrm{D}_{6}, 25^{\circ} \mathrm{C}\right) ; \delta$ $-0.15\left(\mathrm{~s}, 6 \mathrm{H}, \mathrm{Hf}-\mathrm{CH}_{3}\right), 1.05\left(\mathrm{~s}, 18 \mathrm{H},{ }^{\mathrm{B}} \mathrm{Bu}\right), 5.18(\mathrm{t}$, $\left.1 \mathrm{H}, \mathrm{C}_{5} \mathrm{H}_{3}\right), 5.88\left(\mathrm{~s}, 5 \mathrm{H}, \mathrm{C}_{5} \mathrm{H}_{5}\right), 6.21\left(\mathrm{~d}, 2 \mathrm{H}, \mathrm{C}_{5} \mathrm{H}_{3}\right.$ ). ${ }^{13} \mathrm{C}$ NMR (75 MHz, $\left.\mathrm{C}_{6} \mathrm{D}_{6}, 25^{\circ} \mathrm{C}\right): \delta 32.0\left({ }^{\mathrm{B}} \mathrm{Bu}\right), 32.6$ ( $\left.\mathrm{C}_{\text {ipso }}{ }^{\mathrm{B}} \mathrm{Bu}\right), 38.2\left(\mathrm{CH}_{3}\right), 110.5\left(\mathrm{C}_{5} \mathrm{H}_{5}\right), 111.4,111.5$ $\left(\mathrm{C}_{5} \mathrm{H}_{3}\right), 145.0\left(\mathrm{C}_{i p s o} \mathrm{C}_{5} \mathrm{H}_{3}\right)$. Electron impact mass spectrum (70 eV): $m / z=437(31 \%)\left[\mathrm{M}-\mathrm{CH}_{3}\right]^{+}$.

3.7. Preparation of $\left[\left\{\mathrm{H} f\left(\mathrm{C}_{5} \mathrm{H}_{3}-1,3-\mathrm{B}^{\prime} \mathrm{Bu}\right)\left(\mathrm{C}_{5} \mathrm{H}_{5}\right)\left(\mathrm{CH}_{2}\right.\right.\right.$ $\left.\left.\left.\mathrm{C}_{6} \mathrm{H}_{5}\right)\right\}_{2}(\mu-\mathrm{O})\right](7)$

$0.25 \mathrm{~g}(0.73 \mathrm{mmol})$ of $\mathrm{MgBz}_{2} \cdot 2$ THF was added to a solution of $0.3 \mathrm{~g}(0.61 \mathrm{mmol})$ of $\left[\mathrm{Hf}\left(\mathrm{C}_{5} \mathrm{H}_{3}-1,3-\right.\right.$ ' $\left.\mathrm{Bu}_{2}\right)\left(\mathrm{C}_{3} \mathrm{H}_{5}\right) \mathrm{Cl}_{2}$ ] in wet hexane $(50 \mathrm{ml})$ cooled to $-78^{\circ} \mathrm{C}$. The reaction mixture was slowly warmed to room temperature and stirred for $24 \mathrm{~h}$. A yellow solution was formed. After filtration, the solvent was evaporated to dryness to give a yellow solid. Recrystallization from toluene/hexane at $-30^{\circ} \mathrm{C}$ gave a yellow microcrystalline solid which was characterized as $7(0.45 \mathrm{~g}$, $86 \%$ yield).

Anal. Found: $\mathrm{C}, 57.96 ; \mathrm{H}, 6.71 . \mathrm{C}_{30} \mathrm{H}_{66} \mathrm{OHf}_{2}$. Calc.: C, 57.58; H, 6.33\%. 'H NMR (300 MHz, $\left.C_{6} \mathrm{D}_{6}, 25^{\circ} \mathrm{C}\right)$ : \& $1.05\left(\mathrm{~s}, 18 \mathrm{H},{ }^{\prime} \mathrm{Bu}\right), 1.15\left(\mathrm{~s}, 18 \mathrm{H},{ }^{\prime} \mathrm{Bu}\right), 2.19$ (d, 2H, $\left.\mathrm{CH}_{2}\right), 2.20\left(\mathrm{~d}, 2 \mathrm{H}, \mathrm{CH}_{2}\right), 5.59\left(\mathrm{~m}, 2 \mathrm{H}, \mathrm{C}_{5} \mathrm{H}_{3}\right), 5.85(\mathrm{~s}$, $\left.10 \mathrm{H}, \mathrm{C}_{3} \mathrm{H}_{5}\right), 5.99\left(\mathrm{~m}, 2 \mathrm{H}, \mathrm{C}_{3} \mathrm{H}_{3}\right), 6.00\left(\mathrm{~m}, 2 \mathrm{H}, \mathrm{C}_{3} \mathrm{H}_{3}\right)$, $6.93,7.18,7.32(\mathrm{Ph}) .{ }^{13} \mathrm{C}$ NMR (75 MHz, $\left.\mathrm{C}_{6} \mathrm{D}_{6}, 2^{\circ} \mathrm{C}\right)$; $831.2(\mathrm{Bu}), 32.2\left(\mathrm{C}_{i p s v} \mathrm{Bu}\right), 61.7\left(\mathrm{CH}_{2}\right), 105.4$,
116.6, 122.6 $\left(\mathrm{C}_{5} \mathrm{H}_{3}\right), 113.6\left(\mathrm{C}_{5} \mathrm{H}_{5}\right), 114.6,128.1,128.3$, $153.1(\mathrm{Ph}), 136.5,137.2\left(\mathrm{C}_{\text {ipso }} \mathrm{C}_{5} \mathrm{H}_{3}\right)$.

\section{Acknowledgment}

Financial support for this research by DGICYT (Project PB92-0178-C) is gratefully acknowledged.

\section{References}

[1] G. Wilkinson, F.G.A. Stone and E.W. Abel (eds.), Comprehensive Organometallic Chemistry II, Pergamon, Oxford, 1994.

[2](a) P. Jutzi and A. Seufert, J. Organomet. Chem., 169 (1979) 373. (b) P. Jutzi and M. Kuhn, J. Organomet. Chem., 173 (1979) 221. (c) F.R.W.P. Wild, J. Zsolnai, G. Huttner and H.H. Brintzinger, J. Organomet. Chem., 232 (1982) 233. (d) M.D. Raush, J.F. Lewison and W.P. Hart, J. Organomet. Chem., 358 (1988) 161. (e) K.K. Anderson and M. Lin, Organometallics, 7 (1988) 2285. (f) D. Morcos and W.R. Tikkanen, J. Organomet. Chem., 371 (1989) 15. (g) R.L. Halterman, Chem. Rev., 92 (1992) 965. (h) S. Ciruelos, T. Cuenca, P. Gómez-Sal, A. Manzanero and P. Royo, Organometallics, 14 (1995) 177.

[3] (a) R.A. Howie, G.P. McQuillan and D.W. Thompson, J. Organomet. Chem., 268 (1984) 148. (b) I.F. Urazowski, V.I. Ponomaryev, O.G. Ellert, I.E. Nifant'ev and D.A. Lemenovski, J. Organomet. Chem., 356 (1988) 182. (c) I.F. Urazowski, V.I. Ponomaryev, I.E. Nifant'ev and D.A. Lemenovski, J. Organomet. Chem., 368 (1989) 287. (d) I. Jibril, S. Abu Orabi, S.A. Klaib, W. Imhof and G. Huttner, J. Organomet. Chem., 433 (1992) 253. (e) I. Jibril, S. Abu Orabi, S.A. Klaib, L. Zsolnai and G. Huttner, J. Organomet. Chem., 467 (1994) 189. (f) C. Legrand, P. Meunier, J. Petersen, P. Tavares, J. Bodiguel, B. Gautheron and G. Dousse. Organomerallics, 14 (1995) 162 (g) J.I. Amor, T. Cuenca, M. Galakhov and P. Royo, J. Organomet. Chem., 497 (1295) 127.

[4] J.I. Amor, T. Cuenca and P. Royo, unpublished results, 1994.

[5] R.R. Schrock, J. Organomet. Chem., 122 (1976) 209.

[6] R.R. Schrock and J.A. Fellman, J. Am. Chem. Sex., 100 (1978) 3359.

[7] :1.G. Brauer, Handbuch der Prapparativen Anorganischen Chemic, Vol. III, F. Enke, Stuttgart, 1981. 